\title{
Ácidos grasos poliinsaturados en la reproducción de peces: algunos Aspectos fisiológicos y endocrinológicos
}

\author{
Polyunsaturated fatty acids in fish reproduction: some \\ Physiological and endocrinological aspects
}

\section{Os ácidos graxos poliinsaturados em reprodução de peixes: alguns aspectos fisiológicos e endocrinológicos}

\author{
Jonny A Yepes-Blandón ${ }^{1}$; Mónica Botero-Aguirre ${ }^{2}$
}

1 Zoot, MSc, (c) PhD; Grupo de investigación en Ciencias Agrarias (GRICA), Escuela de producción Agropecuaria; Facultad de Ciencias Agrarias, Universidad de Antioquia, Grupo de investigación en Peces Nativos (GIPEN), Piscícola San Silvestre S.A., Barrancabermeja Colombia.

2 Zoot, PhD; Grupo de investigación en Ciencias Agrarias (GRICA), Escuela de producción Agropecuaria; Facultad de Ciencias Agrarias, Universidad de Antioquia.

Email: jonny.yepes@udea.edu.co

Recibido: 06 de febrero de 2017

Aceptado: 08 de junio de 2018

\begin{abstract}
Resumen
La FAO, 2012 y 2014 plantea la importancia de la reproducción en la piscicultura. El incremento de la producción mundial de peces se debe a la intensificación de los sistemas de cultivo, lo que significa producir grandes cantidades de alevinos para crecimiento y ceba (Orozco, 2012). En la última década, se ha investigado la influencia de la nutrición en la reproducción, muchos autores plantean que, nutrientes como los ácidos grasos poliinsaturados (AGPI), afectan la reproducción (Tocher, 2010, Turchini et al., 2011). Varios estudios se han centrado en el establecimiento de los requerimientos nutricionales para el éxito reproductivo, sin embargo, sobre AGPI, es poco lo que se ha logrado determinar (Pohlenz et al., 2014). Lo anterior, ha obligado a realizar estudios con herramientas ómicas (Rodrígues et al., 2012), que integren la información ambiental y la genética en el cerebro de los peces para la transducción en señales neuronales a través de mecanismos bioquímicos y fisiológicos, capaces de modular la secreción hormonal implicada en el proceso reproductivo de los peces (Wing-Keong y Wang, 2011; Norambuena et al., 2012). Estos procesos bioquímicos en peces requieren ser dilucidados para mejorar los sistemas de cultivo, aumentar los niveles de producción, satisfacer la demanda y lograr avances impactantes en el campo del conocimiento científico.
\end{abstract}

Palabras clave: Lípidos, reproducción, nutrición, genómica, nutrigenómica

\begin{abstract}
FAO, 2012 and 2014, raises the importance of reproduction in fish farming. The increase in world fish production is due to the intensification of farming systems, which means producing large numbers of fingerlings for growth and fattening (Orozco, 2012). In the last decade, the influence of nutrition on reproduction has been investigated, many authors suggest that nutrients such as polyunsaturated fatty acids (PUFA) affect reproduction (Tocher, 2010, Turchini et al., 2011). Several studies have focused on the establishment of nutritional requirements for reproductive success, however, few determinations on PUFA, have been made (Pohlenz et al., 2014). Consequently, several studies have used omics tools (Rodrígues et
\end{abstract}


al., 2012), to integrate environmental and genetic information in the fish brain for transduction into neuronal signals through biochemical and physiological mechanisms, capable of modulating the hormonal secretion involved in fish reproduction (Wing-Keong and Wang, 2011, Norambuena et al., 2012). These biochemical processes in fish need to be elucidated to improve farming systems, increase production levels, meet demand and achieve impressive advances in the field of scientific knowledge.

Key words: Lipids, reproduction, nutrition, genomics, nutrigenomics

\section{Resumo}

A FAO, 2012 e 2014, destaca a importância da reprodução na piscicultura. O aumento da produção mundial de peixes deve-se à intensificação dos sistemas de criação, o que significa produzir um grande número de alevinos para crescimento e engorda (Orozco, 2012). Na última década, tem-se pesquisado sobre a influência da nutrição na reprodução. Muitos autores sugerem que nutrientes, como os ácidos graxos poliinsaturados (PUFAs), afetam a reprodução (Tocher, 2010, Turchini et al., 2011). Alguns estudos têm focado seus esforços no estabelecimento de exigências nutricionais para o sucesso reprodutivo. Contudo, são escassos os estudos avaliando os efeitos dos PUFAs sobre aspectos reprodutivos (Pohlenz et al., 2014). Assim, estudos com ferramentas ómicas são relevantes (Rodrigues et al., 2012), ao integrar informação genética e ambiental no cérebro do peixe para a transdução de sinais neuronais através de mecanismos bioquímicos e fisiológicos, modulando a secreção hormonal envolvida no processo reprodutivo dos peixes (Wing-Keong e Wang, 2011, Norambuena et al., 2012). Esses processos bioquímicos nos peixes precisam ser elucidados para melhorar os sistemas de criação, aumentar os níveis de produção, atender à demanda e alcançar avanços científicos significativos.

Palavras chave: Lipídios, reprodução, nutrição, genômica, nutrigenômica

\section{Introducción}

La acuicultura genera un aporte significativo de proteína animal para el consumo humano y las tendencias muestran que lo será más para las generaciones futuras (Tacon y Metian, 2013 y FAO, 2014). En las últimas décadas, la piscicultura ha presentado un crecimiento anual superior al de otras industrias de producción animal (FAO, 2012 y Pohlenz et al., 2014), pasando de 1.7 millones de toneladas en 1957 a 68 millones de toneladas en 2008 (FAO, 2012). En particular, en especies de interés económico como la tilapia, cuya producción mundial se ha elevado a 3 millones de toneladas en el año 2010 (Wing-Keong y Wang, 2011), y se estima que aumentará alrededor de 8,9 millones de toneladas para el año 2020 (Tacón y Metían, 2008; Wing-Keong y Wang, 2011 y FAO, 2014).

En Colombia, la cadena piscícola ha presentado en los últimos años avances en materia de competitividad, mayor viabilidad, potencial de crecimiento económico y generación de empleo y de divisas. Lo anterior, como consecuencia del mejoramiento de los sistemas de cultivo, aumento de los volúmenes de producción, incremento de la oferta de semilla de algunas especies piscícolas, mejora en el índice de conversión alimenticia y reducción en los precios de los peces de cultivo con respecto a otros productos cárnicos. De igual manera, por las características geográficas del país en cuanto a disponibilidad de recursos hídricos y de suelos aptos para esta actividad (Suárez, 2011).
Por otro lado, las exportaciones colombianas a los Estados Unidos representadas principalmente en filete fresco de tilapia aumentaron $212,3 \%$ en el 2008. En el 2011 se produjeron 3272 toneladas de filete, con respecto a las 1108 toneladas producidas en 2007 (Trademap, 2012), constituyéndose en la principal especie piscícola producida en el país (58\%) $(\mathrm{CCl}$, 2009), donde se destacan como principales productores los departamentos de Huila, Meta y Antioquia (Orozco, 2012). Este rápido crecimiento ha dado lugar al aumento de la demanda de alevinos que satisfagan la oferta (Izquierdo et al., 2001 y Orozco, 2012).

A pesar de los avances en la producción piscícola, no se están satisfaciendo las necesidades del mercado, debido a factores tales como la baja fecundidad de los reproductores, el número de huevos producidos en cada desove y la asincronía de las hembras para la ovoposición. (Constantinos et al., 2010; Bobe y Labbé, 2010). Por lo que se requieren estrategias para mejorar la producción de elevadas cantidades de ovas y de alevinos de excelente calidad y resistencia (WingKeong y Wang, 2011).

Por lo anterior, la nutrición de los peces principalmente, los lípidos y los ácidos grasos poliinsaturados (AGPIs) han sido identificados como nutrientes clave que afectan el desempeño fisiológico de los reproductores (Bell y Sargent, 2003; Watanabe y Vassallo-Agius, 2003; Tocher, 2010, Polakof et al., 2010, Turchini et al., 2011 y NRC, 2011). Aun así, es poca la información relacionada entre los AGPI y la expresión de genes im- 
plicados en la fisiología reproductiva (Jaya-Ram et al., 2008).

\section{La reproducción de los peces}

La reproducción de peces en cautiverio puede ser controlada por manipulaciones ambientales, tales como el fotoperíodo, la temperatura del agua o el sustrato de desove. Sin embargo, la biología de algunos peces no es muy conocida o incluso son imposibles de simular los parámetros ambientales necesarios para el desempeño reproductivo natural. En estos casos, existen otras formas de mejorar la reproducción tales como: el uso de hormonas y la nutrición (Constantinos et al., 2010).

El uso de hormonas exógenas es una manera eficaz para inducir la maduración reproductiva. Se pueden usar como herramientas para mejorar la eficiencia de la producción de huevos, aumentar la espermiación y facilitar los procesos de incubación (Constantinos et al., 2010). Agulleiro et al., (2007), Guzmán et al., (2009), Carazo et al., (2011) y Norambuena et al., (2012) afirman que el uso de terapias hormonales aumenta el número y el volumen de huevos, pero no se mejora el desempeño reproductivo y la calidad. Por otro lado, la disfunción de los reproductores podría estar determinada por factores ambientales en etapas tempranas del desarrollo, factores abióticos y/o efectos de la selección de reproductores y en particular la nutrición (Mañanos et al., 2008 y Howell et al., 2011).

Desde hace varios años, el campo de la nutrición de peces se ha centrado principalmente en el establecimiento de los requerimientos mínimos para el desarrollo, el crecimiento y el éxito reproductivo de las diferentes especies (NRC, 2011 y Pohlenz et al., 2014). Estudios realizados por Izquierdo et al., (2001), Watanabe y Vassallo-Agius, (2003), Ling et al., (2006), Hachero-Cruzado et al., (2009) y Norambuena et al., (2012), demostraron que numerosos factores tales como la ración y la composición del alimento, influyen en parámetros reproductivos tales como el desarrollo gonadal, la cantidad y la calidad de los huevos, el éxito del desove, el porcentaje de eclosión y la calidad de las larvas producidas, debido a que se afecta la disponibilidad de los componentes bioquímicos esenciales para la gametogénesis y el control de la reproducción (Izquierdo et al., 2001 y Norambuena et al., 2012).

En la actualidad, la información sobre los requerimientos nutricionales de reproductores de especies como la tilapia, aún es escasa, especialmente en relación con lípidos (El-Sayed et al., 2008 Lupatsch et al., 2010).
Los lípidos, principalmente AGPIs requeridos en la dieta pueden ejercer su influencia sobre las actividades de desaturación y elongación, directamente a través de la modificación de la fluidez de la membrana celular o indirectamente a través de la regulación de diversos factores esenciales para la activación o represión de genes tanto de desaturasas como de elongasas importantes para la síntesis de los ácidos grasos esenciales (AGEs) (Jump et al., 1999; Jump 2002 Jaya-Ram 2008). También son importantes para otras funciones como el control de la reproducción y el desarrollo de embriones y/o larvas de peces (Izquierdo et al., 2001 y Norambuena et al., 2012). Dentro de los AGPIs, los de mayor importancia son el ácido linolénico (18:3 $\omega$-3, ALN) y el ácido linoleico (18:2 $\omega-6$, AL) también conocidos como omega-3 y omega-6 respectivamente. Ambos ácidos grasos son considerados como AGEs, debido a la incapacidad en los peces de sintetizarlos en el organismo, debiendo ser proporcionados en la dieta (Izquierdo et al., 2001 y Norambuena et al., 2012).

Estudios realizados por Furuita et al., (2003), Meunpol et al., (2005), Pérez et al., (2008) y Tocher, (2010), han demostrado que AGPIs suministrados en la dieta de peces dulceacuícolas, intervienen en la síntesis otros AGEs como el ácido araquidónico (20:4 n-6, AA), el ácido eicosapentaenoico (20:5 n-3, AEP) y el ácido docosahexaenoico (22:6 n-3, ADH), los cuales, tienen efectos positivos sobre la sobrevivencia y la calidad de la descendencia (Izquierdo et al., 2001; Watanabe y Vassallo-Agius, 2003; Muskiet et al., 2006; Pickova et al., 2007; Pérez et al., 2008; El-Sayed y Kawanna, 2008; Sawanboonchun, 2009; Lupatsch et al., 2010; Tocher, 2010; Navarro et al., 2010a; Norambuena et al., 2012 y Morais et al., 2014), además, de ser una fuente de energía metabólica que se almacena principalmente en hígado y músculo (Tocher, 2003; Cejas et al., 2004 y Norambuena et al., 2012).

Por otro lado, los AGEs, mediante la ciclación e incorporación de oxígeno molecular, actúan como precursores de los eicosanoides, un grupo de moléculas de 20 átomos de carbono de carácter lipídico, por la vía de la enzima ciclooxigenasa, tiene como metabolitos resultantes la prostanglandina $\mathrm{E}_{2}$, la prostaciclina y los tromboxanos, y por la vía lipooxigenasa da lugar a productos como leucotrienos y a ciertos hidroxiácidos (Izquierdo et al., 2001; Watanabe y Vassallo-Agius, 2003; Pickova et al., 2007; Sawanboonchun, 2009; Simopoulos, 2011 y Janssen et al., 2014).

Los AGEs son considerados como un grupo fisiológicamente dinámico de moléculas responsables de una amplia variedad de actividades celulares incluyendo la 
regulación de genes, la señalización y el mantenimiento de la integridad de la membrana celular (Furuita et al., 2003 y Navarro et al., 2010). Además, de contribuir con el buen funcionamiento del sistema neural (Bell et al., 1995 y Norambuena et al., 2012), ayudan al desarrollo del cerebro y de los ojos (Brodtkorb et al., 1997 y Norambuena et al., 2012), al igual que son constituyentes de las moléculas involucradas en las redes más complejas de comunicación celular del organismo animal (Izquierdo et al., 2001; Watanabe y Vassallo-Agius, 2003; Pickova et al., 2007 y Sawanboonchun, 2009). Por otro lado, participan en la síntesis de los esteroides gonadales (andrógenos, estrógenos y progestágenos) importantes en los procesos reproductivos (Wade et al., 1994 y Norambuena et al., 2012).

Si bien, todos los estudios e información antes mencionados sugieren que los AGPIs de la dieta intervienen en la regulación de la reproducción de peces teleósteos, los mecanismos reales y vías involucradas aún no se conocen (Jaya-Ram et al., 2008). Por ello, es necesario comprender en detalle el papel real de los AGPIs en el proceso de la reproducción de los peces (Bell y Sargent, 2003, Subhadra et al., 2006; Karapanagiotidis et al., 2007; Teoh et al., 2011 y Wing-Keong et al., 2013).

Autores como Izquierdo et al., (2001), Bell y Sargent, (2003), Furuita et al., (2007), Wilson, (2009) y Tocher, (2010), afirman que proporciones en la dieta de ADH/ AEP y AEP/AA pueden influir en la calidad de los huevos y de las larvas de muchas especies. De igual manera se ha reportado un efecto directo de la composición de ácidos grasos en la dieta de los reproductores sobre la composición de ácidos grasos en los huevos (Tocher, 2010). Estudios realizados por Vassallo-Agius et al., (2001) en reproductores de trucha arcoíris (Oncorhynchus mykiss), Mazorra et al., (2003) en fletán (Hippoglossus hippoglossus), Furuita et al., (2003) en lenguado japonés (Paralichthys olivaceus), Ling et al., (2006) en pez espada (Xiphophorus helleri), Furuita et al., (2007) en anguila (Anguilla japónica) y Lund et al., (2008) en lenguado común (Solea solea) reportaron que un mínimo de 1,5 a 2,0\% de AGPIs en la dieta son necesarios para garantizar huevos y larvas de alta calidad.

En acuicultura, para garantizar el aporte de AGPIs en la dieta se han utilizado tradicionalmente los aceites de peces marinos. Se estima que se consume el $90 \%$ de la oferta mundial para la elaboración de alimentos balanceados debido a la expansión de esta industria, poniendo en riesgo las necesidades del mercado (Tacon y Metian, 2008, Turchini et al., 2009, Jackson y Shepherd, 2012 y Shepherd y Jackson, 2013). Por lo tanto, se hace necesario explorar nuevas alternativas para reemplazarlos, debido a la disminución de la oferta y al incremento en los costos de producción de alimentos para las especies acuícolas (Sorbera et al., 2001 y Turchini et al., 2009).

Los aceites vegetales se presentan como una alternativa viable y más rentable en comparación con aceites de pescados marinos (APM). Varios estudios han reportado que los aceites vegetales pueden reemplazar parcial o completamente los APM en las dietas de peces sin comprometer el rendimiento, el crecimiento y los demás procesos fisiológicos, esto siempre y cuando los requerimientos de AGEs no se descompensen y se conserven las proporciones de éstos en los alimentos balanceados ofrecidos (Bell et al., 2003, FonsecaMadrigal et al., 2005, Karapanagiotidis et al., 2007, Turchini et al., 2009, Teoh et al., 2011 y Wing-Keong et al., 2013). Sin embargo, la información sobre el efecto de los aceites vegetales en relación con el desempeño reproductivo de los peces es muy poca (Wing-Keong y Wang, 2013).

Los requerimientos de AGEs varían según las especies piscícolas así; las grasas de los peces se caracterizan por su elevada proporción de ácidos grasos insaturados, tanto marinos como dulceacuícolas presentan una composición muy variable, resultando en una alta proporción de $\omega-3$, que sobrepasa a los $\omega-6$. La proporción $\omega-6 / \omega$-3 es en peces dulceacuícolas de 0.37 $(1: 2.77)$, en peces marinos es de 0.16 (1:6.25) (Tocher et al., 2002; Seiliez et al., 2003; Karapanagiotidis et al., 2007; Teoh et al., 2011 y Wing-Keong et al., 2013). Aunque, es sabido que los peces de agua dulce usan más el ácido linoleico y los marinos más el ácido linolénico.

\section{Aspectos endocrinos involucrados en la reproducción de peces}

La reproducción de los peces puede ser más eficiente al controlar y modificar la secreción hormonal de la hipófisis a través del conocimiento de la función del eje cerebro-hipófisis-gónadas (CHG) y el papel de las gonadotropinas en la reproducción (Levavi-Sivan et al., 2010).

El inicio de la maduración sexual en peces presenta dos eventos simultáneos: la función del eje $\mathrm{CHG}$, influenciado por la hormona liberadora de la gonadotropina (GnRH) (Zanuy et al., 2001; Okuzawa, 2002; Weltzien et al., 2004; Jalabert, 2005; Dufour y Rousseau, 2007 y Taranger et al., 2010), y la activación de sus receptores en las gónadas para la producción de células germinales y la síntesis de esteroides sexuales requeridos para el desarrollo de las células germinales 
en hembras (estrógenos y vitelogénesis), en machos (andrógenos y espermatogénesis), progestágenos y factores de crecimiento que son los efectos finales del desarrollo gonadal (Schulz et al., 2010).

\section{Hormona liberadora de gonadotropinas (GnRH).}

Igual que en mamíferos, la hipófisis de los teleósteos adultos se encuentra en una cámara ósea, posterior al quiasma óptico, justo por encima del hueso esfenoides situado por debajo del hipotálamo y conectado por un tallo corto (Frisen, 1967 y Levavi-Sivan B et al., 2010). En los peces dicha cámara ósea, se compone de fibras neurosecretoras que pasan desde el cerebro a la hipófisis, compuesta por la adenohipófisis y la neurohipófisis. La primera contiene las diferentes células secretoras de las hormonas de la hipófisis y por lo tanto representa la parte glandular de la misma. La neurohipófisis se compone principalmente de haces de fibras neurosecretoras procedentes de diferentes partes del cerebro y secretoras de diversos péptidos (Chapman et al., 2005; Pogoda y Hammerschmidt, 2007 y Zohar et al., 2010).

Desde el aislamiento de $\mathrm{GnRH}$, se encontró que la estructura del gen que la codifica fue altamente conservada durante la evolución de los vertebrados y se compone de 4 exones y 3 intrones (Hildahl et al., 2011). La secuencia de codificación transcribe una proteína precursora de 92 aminoácidos (aa) que origina la GnRH y un péptido de 56 aa llamado GAP (péptido asociado con GnRH). El primer exón consiste en una región $5^{\prime}$ no transcrita que difiere en los ADN codificados del hipotálamo; el segundo exón codifica para el péptido señal, GnRH de 10 aa y los primeros 11 residuos de aa de GAP; el tercer exón codifica para los residuos 12 al 43 de GAP y el cuarto exón codifica para los 13 residuos de aa terminales de GAP y el restante es mRNA que no se transduce (Roch et al., 2011).

Así mismo, en varios estudios se ha reportado, que esta molécula se ha conservado desde los peces hasta el ser humano (Counis et al., 2005). Además, muestran que en vertebrados existen por lo menos cinco variantes (Counis et al., 2005 y Hildahl et al., 2011). Otras variantes fueron descubiertas más tarde en los vertebrados no mamíferos y se han visto involucradas en el control del comportamiento reproductivo (Ogawa et al., 2006; Volkoff et al., 2009 y Hildahl et al., 2011). Estudios más recientes sugieren que la GnRH está implicada en el desarrollo de las neuronas, la neuromodulación y el desempeño reproductivo (Ogawa et al., 2006; Palevitch et al., 2009 y Hildahl et al., 2013).
Por lo anterior ahora es sabido que diversas especies, contienen dos o más isoformas de GnRH. Por ejemplo, los peces teleósteos poseen tres genes que muestran distintos patrones de expresión en el cerebro (Zohar et al., 2010).

Los genes identificados en los peces teleósteos son: GnRH1 que es una forma específica de las especies de $\mathrm{GnRH}$ expresada y localizada principalmente en el bulbo olfativo, telencéfalo ventral y la zona preóptica (González-Martínez et al., 2002). GnRH2, que es una forma conservada desde los peces hasta los mamíferos, fue identificada por primera vez en el poIlo (cGnRH-II) (Miyamoto et al., 1982 y Hildahl et al., 2011) y se encuentra principalmente en el tegmento del mesencéfalo. Por último, la GnRH3, que es una forma específica de la GnRH en los peces, se identificó originalmente en el salmón (sGnRH) (Sherwood et al., 1983 y Hildahl et al., 2011) y tiene distribución similar a GnRH1.

La diversificación funcional de estas hormonas no se entiende completamente, pero la principal hormona hipofisiotrópica en la mayoría de los teleósteos se considera que es GNRH1, debido a su expresión en el prosencéfalo ventral y a la estimulación de las principales gonadotropinas (FSH y LH). También, se ha reportado que la GnRH1 estimula la síntesis de otras hormonas de la hipófisis en algunas especies, tales como, la hormona de crecimiento $(\mathrm{GH})$, la prolactina (PRL), la adrenocorticotropa (ACTH) y la somatolactina (SL), las cuales son permisivas y regulan otros sistemas fisiológicos que indirecta, pero profundamente, influencian la reproducción (Yaron et al., 2003; Aizen et al., 2007 y Constantinos et al., 2010). La molécula de la GnRH, puede sufrir cambios conformacionales que le permiten ir desde una forma completamente extendida hasta una forma altamente enrollada (Constantinos et al., 2010). Además, el análisis tridimensional de la hormona ha indicado que existe un giro B en la posición 6, que aproxima el carboxilo a la amina terminal, dicha configuración tiene una alta afinidad por los receptores de GnRH. (Carolsfeld et al., 2000 y Hildahl et al., 2011).

Debido a esto, se le atribuye a la GnRH como función principal la estimulación para la síntesis y liberación de las hormonas gonadotrópicas o GtHs en teleósteos (Imanaga et al., 2014), como en todos los vertebrados permitiendo de este modo la regulación del eje $\mathrm{CHG}$, además su estimulación conduce a la gametogénesis y a la producción de esteroides en las gónadas (Roch et al., 2011) 
Ácidos grasos, posibles reguladores de las hormonas gonadotrópicas

En peces al igual que en los mamíferos, el hipotálamo regula la liberación de hormonas de la hipófisis tales como las gonadotropinas a través de varias neurohormonas asociadas al eje $\mathrm{CHG}$ como la $\mathrm{GnRH}$, el GABA (ácido gamma-aminobutírico), la PACAP (polipéptido activador de adenilato ciclasa de la hipófisis), el IGF-I, la norepinefrina, el NPY (neuropéptido Y), la kisspectina, la leptina y la ghrelina y el inhibidor de GnRH la dopamina. Además, los esteroides gonadales y péptidos ejercen sus efectos sobre éstas, ya sea directamente o a través del hipotálamo (Levavi et al., 2010).

La acción de dichas neurohormonas requiere la unión a receptores específicos anclados en la membrana plasmática de las células diana de las gónadas, donde activan una o más vías de segundos mensajeros, que conducen a la secreción de esteroides sexuales (estradiol en hembras y 11-cetotestosterona (11 -KT) en los machos) (Levavi et al., 2010).

Moberg et al., (1995), demostraron la existencia de dos gonadotropinas en peces, las Ilamaron GtH-I y GtH-II, estructuralmente las GTHs al igual que las de los mamíferos, son glicoproteínas heterodiméricas que en una misma especie comparten una subunidad $\alpha$ común unida de forma no covalente a una subunidad $\beta$ específica la cual determina la especificidad biológica de la hormona (Yaron et al., 2003). Cada subunidad, $\alpha$ FSH $\beta$ y LH $\beta$ está codificada por un gen diferente en su secuencia de aminoácidos y conservación filogenética. El heterodímero completo $\alpha / \beta$ es capaz de unirse específicamente a su receptor, pero cada subunidad por separado es inactiva (Yaron e et al., 2003). Querat et al., (2000), clonaron las subunidades $\alpha / \beta$ de GtH-I y GtH-II y basados en su árbol filogenético, sugirieron que se deben denominar $\mathrm{FSH} \beta$ y $\mathrm{LH} \beta$ respectivamente.

Las GtHs FSH $\beta$ y LH $\beta$ son producidas en las células gonadotropas de la adenohipófisis (Weltzien et al., 2014), gracias a la hormona $\mathrm{GnRH}$ de las neuronas del hipotálamo se estimula su síntesis y liberación para después unirse a sus receptores específicos, FSHR y LHR en las gónadas, estimulando la producción de gametos masculinos y femeninos (Weltzien et al., 2004, Rocha et al., 2007 y Mittelholzer et al., 2009).

La liberación de las dos GHTs son reguladas diferencialmente durante el ciclo reproductivo, pero los detalles de los mecanismos detrás de esta regulación diferencial aún están lejos de ser claros (Padmanabhan y McNeilly, 2001 y Sharma et al., 2012). Los avances recientes indican un control diferencial de la transcripción de estos genes por factores paracrinos, así como efecto diferencial de pulsos de frecuencia de la GnRH (Bernard et al., 2010; Thackray et al., 2010; Levavi et al., 2010; Sharma et al., 2012; Thompson y Kaiser, 2014; Hodne et al., 2013).

Aunque Moberg et al., (1995); Planas y Swanson, (1995) y Levavi et al., (2010) realizaron algunos estudios en especies marinas como el salmón y expusieron que las concentraciones plasmáticas y en la pituitaria de $\mathrm{FSH} \beta$ eran mayores que $\mathrm{LH} \beta$ durante la vitelogénesis y en estadios iniciales de la espermatogénesis; y los niveles de LH $\beta$ eran mayores durante la ovulación y la espermiación debido a su potente acción sobre la estimulación de $17 \alpha$, 20ß-dihidroxi-4-pregnen-3-ona (DHP) en las etapas finales de la maduración de ovocitos, la ovulación en las hembras y la espermiación en los machos (Planas y Swanson, 1995; Yaron et al., 2003; Levavi-Sivan et al., 2010 y Aizen et al., 2012). Lo anterior ha permitido a varios investigadores concluir que los peces poseen un sistema doble de $\mathrm{GtH}$ s equipotentes que controlan la reproducción (Swanson et al., 1991 y Levavi-Sivan et al., 2010).

Los AGPI, mantienen niveles elevados de hormonas gonadotrópicas (FSH $\beta$, LH $\beta$ ) (Izquierdo, 2001). Achata, (1995) y Gulliver et al., (2012), explican una de las vías por la cual se manifiesta el aumento de las concentraciones de $\mathrm{FSH} \beta$ y $\mathrm{LH} \beta$ a partir de la suplementación con AGPI en mamíferos. Estos autores afirman que la mayoría de los ácidos grasos de la dieta son incorporados dentro de los fosfolípidos, posteriormente con un estímulo apropiado, la fosfolipasa A es activada trayendo consigo la liberación de los ácidos grasos esenciales, especialmente el AA, que inicia la formación de las prostaglandinas (PGs).

Sin embargo, autores como Aranda et al., (2002), proponen un posible mecanismo de cómo intervienen los lípidos en la reproducción de mamíferos. A partir de pregnenolona, primera hormona esteroidea derivada del colesterol y precursora de progesterona, se da origen a corticoesteroides y a hormonas sexuales (estrógenos). Al aumentar las concentraciones de estrógenos se eleva la producción de $\mathrm{LH} \beta$ y FSH $\beta$ por estímulo en la adenohipófisis. Además, los estrógenos actúan en forma sinérgica con las gonadotropinas promoviendo crecimiento ovárico, formación de receptores de $\mathrm{LH} \beta$ y FSH $\beta$ y actividad misma de la aromatasa, lo que explicaría el aumento preovulatorio de los niveles circulantes de estradiol. Estas hormonas también podrían desempeñar un papel central en el proceso de selección y dominancia folicular (Mateos, 1999). Por otro lado, en estudios más recientes de Gulliver et al., (2012) se proponen varios mecanismos que se deben 
considerar al momento de examinar el efecto y vías de los AGPIs.

Estudios realizados en otras especies, reportan que dietas enriquecidas con ácidos grasos, influyen sobre la fertilidad afectando diversos elementos del eje CHG (Wathes et al., 2007). Por ejemplo, la inclusión de AGPIs a ratas in vivo aumenta la expresión de LH $\beta$ (Garrel et al., 2011) y afecta la gametogénesis. La composición de AGPIs de las membranas de las células de los espermatozoides y de los ovocitos son importantes durante la fertilización (Aitken y Baker, 1995). Las células de Leydig y de Sertoli son importantes para el metabolismo de lípidos en machos, participan en la regulación del nivel de colesterol necesario en la producción de esteroides que aseguren la composición apropiada de la membrana de los espermatozoides y garanticen los fosfolípidos para mantener la calidad de los mismos (Simopoulos, 2011, Dunning y Robker, 2012 y Janssen et al., 2014).

La acumulación de pruebas indica que la $\beta$-oxidación de los ácidos grasos en las hembras es esencial para la maduración de ovocitos y durante las primeras etapas del desarrollo del embrión, también son precursores de las prostaglandinas y esteroides, importantes en múltiples funciones reproductivas (Dunning y Robker, 2012). Autores como Hachero et al., (2013) estudiaron los resultados de la inactivación de los genes implicados en el metabolismo de lípidos (por ejemplo, LXR) o en el flujo de colesterol, encontrando que conducen a subfertilidad o esterilidad.

\section{Regulación endocrina: ciclo reproductivo de las hembras de tilapia}

En los últimos años, la tilapia se ha convertido en uno de los peces más importante en la acuicultura mundial. Esta especie se considera apropiada por las potenciales características de crecimiento, intervalo entre desoves, facilidad de desove, resistencia a enfermedades, eurihalina, soportar tenores bajos de oxígeno, altas densidades de siembra, buena rusticidad y buena calidad nutricional (Coward y Little, 2001, Matan et al., 2013 y Corinne et al., 2014). Estas características, adicionales al genoma recientemente publicado, hacen de la tilapia un modelo cada vez más popular para los estudios evolutivos, fisiológicos y desempeño productivo (Matan et al., 2013 y Corinne et al., 2014).

En la tilapia hay dos tipos de receptores de GnRH (GnRHR): GnRHR3 es el tipo de receptor para la reproducción, mientras que GnRHR1 es probablemente el tipo de receptor que se expresa en los somatotrofos, que son las células de la hipófisis que producen la hormona del crecimiento $(\mathrm{GH})$, y responden a la liberación de ésta, en respuesta a la estimulación de la hormona liberadora de la hormona del crecimiento $(\mathrm{GHRH})$, o a la inhibición de la somatostatina (Chen y Fernald, 2008).

Durante la vitelogénesis, las hembras de tilapia generalmente presentan aumento de los niveles de RNAm en GnRHR3 y GnRHR1 como parte de la retroalimentación positiva sobre la liberación de FSH $\beta$, aumento de los receptores de dopamina D2 (DRD2) y aumento de las concentraciones de estradiol $\left(E_{2}\right)$ (Levavi-Sivan et al., 2003; Levavi-Sivan et al., 2005) e inhibición de la liberación de FSH $\beta$ y LH $\beta$ y síntesis de LH $\beta$ como parte de la retroalimentación negativa. Al final de la vitelogénesis, hay un aumento en la síntesis y liberación de FSH $\beta$, como parte de la preparación para una nueva generación de folículos (Aizen et al., 2007, LevaviSivan et al., 2006 Levavi et al., 2010).

En la maduración, las etapas incluyen: (1) aumento en los niveles de RNAm de GnRHR3 (Levavi-Sivan et al., 2004); (2) aumento en los niveles de DRD2, lo que probablemente refleja la retroalimentación negativa sobre la liberación de LH; (3) disminución en los niveles de RNAm de DRD2 concomitantes con el aumento de la GnRHR3, probablemente como parte del reclutamiento de una nueva generación de ovocitos para un nuevo ciclo; (4) aumento en la síntesis de LH $\beta$ en dosis más bajas de DHP (hacia la maduración final de los oocitos) y disminución en dosis más altas de DHP, probablemente como parte de la retroalimentación negativa, y (5) aumento en la síntesis de FSH $\beta$ en dosis más bajas de DHP como parte del reclutamiento de una nueva generación de ovocitos para un nuevo ciclo (Levavi-Sivan et al., 2006).

La síntesis y la liberación de hormonas de la reproducción en diferentes etapas de las hembras de tilapia, como modelo para estudio de la fisiología reproductiva, presentan dos picos claros de ambas gonadotropinas $(\mathrm{FSH} \beta$ y $\mathrm{LH} \beta)$, en la vitelogénesis y en la maduración. El primero es evidente 2-3 días después del desove, en la fase de vitelogénesis. Los niveles relativamente altos de $\mathrm{LH} \beta$ durante la fase de vitelogénesis son concomitantes con el aumento de la FSH $\beta$; por ello en tilapia se sugiere que la LH $\beta$ juega un papel no sólo durante la maduración final de los oocitos, sino también durante la vitelogénesis. El segundo pico es evidente justo antes del siguiente desove. El aumento de la FSH $\beta$ probablemente refleja el perfil hormonal durante el reclutamiento de una nueva generación de folículos para el próximo ciclo (Aizen et al., 2007 y Levavi-Sivan et al., 2010). En la tilapia, el nivel de $17 \beta$-estradiol, alcanza su punto máximo cuando se produce la vitelogénesis y los niveles de DHP aumen- 
tan de forma similar con los niveles de LH $\beta$, lo que sugiere su participación en eventos de maduración final (Tacon et al., 2000 y Levavi-Sivan et al., 2010).

Partiendo de la biología reproductiva de la tilapia y apoyados en las investigaciones realizadas en múltiples especies piscícolas, se hace necesario indagar y estudiar con detalle el género Oreochromis para identificar la participación de los AGPIs en cuanto a producción de ovas y a frecuencia reproductiva, a través de la relación que tienen sobre los genes participantes en el proceso reproductivo y la identificación de la posible ruta metabólica.

Lo anterior con el objetivo de contribuir a la producción de semilla de tilapia con estándares de calidad, a través de una dieta rica en ácidos omega 3 y omega 6 que permitan mejorar su desempeño productivo y reproductivo (Simopoulos, 2011; Janssen et al., 2014). et al., 2014). Por otro lado, es importante el desarrollo de investigaciones que permitan dilucidar la ruta metabólica y los mecanismos por cuales los ácidos grasos poliinsaturados ejercen su función en la reproducción de la tilapia, mediante el uso de herramientas tecnológicas avanzadas como la proteómica.

\section{Referencias}

Achata Böttger J. 1995. Principios de bioquímica. Articulo prostaglandinas, Lehninger. Editorial Omega. España segunda edición.

Agulleiro MJ, Scott AP, Duncan N, Mylonas CC, Cerdá J. Treatment of GnRHa-implanted Senegalese sole (Solea senegalensis) with 11-ketoandrostenedione stimulates spermatogenesis and increases sperm motility. Comp Biochem Physiol A Mol Integr Physiol. 2007;147(4):885-892.

Aizen J, Harel J, Levavi-Sivan B. Development of specific enzymelinked immunosorbent assay for determining $\mathrm{LH}$ and FSH levels in tilapia, using recombinant gonadotropins. Gen Comp Endocrinol. 2007;153: 323-332.

Aizen J, Kobayashi M, Selicharova I, Chang Sohn Y, Yoshizaki G, Levavi-Sivan B. Steroidogenic response of carp ovaries to piscine FSH and LH depends on the reproductive phase. Gen Comp Endocrinol. 2012;178: 28-36.

Aranda MV, Brave N, Casagrande R. 2012. Colesterol en bovinos. Sitio Argentino de Producción Animal. [Internet]. Disponible en: http:// www.produccionanimal.com.ar/informacion_tecnica/ carne_y_subproductos/26colesterol_en_bovinos.pdf.

Bell JG Sargent JR. Arachidonic acid in aquaculture feeds: current status and future opportunities. Aquaculture. 2003;218:491499 .

Bell M, Batty R, Dick J, Fretwell K, Navarro J, Sargent J. Dietary deficiency of docosahexaenoic acid impairs vision at low light intensities in juvenile herring (Clupea harengus L) Lipids. 1995;30:443-449.
Bernard DJ, Fortin J, Wang Y, Lamba P. Mechanisms of FSH synthesis: what we know, what we don't, and why you should care. Fertil Steril. 2010;93:2465-2485.

Bobe J Labbé C. Egg and sperm quality in fish. Gen Comp Endocrinol. 2010;165:535-548.

Brodtkorb T, Rosenlund G, Lie. Effects of dietary levels of 20:5n $\square 3$ and 22:6n - 3 on tissue lipid composition in juvenile Atlantic salmon, Salmo salar, with emphasis on brain and eye. Aquacult Nutr. 1997;3:175-187.

Carazo I, Martin O, Chereguini E, Mañanós N, Duncan. 2011. The absence of reproductive behaviour in cultured ( $\mathrm{G} 1$ generation) Senegalese sole (Solea senegalensis) explains poor reproductive performance proceedings of 5 th Workshop on the. Cultivation of Soles, Centre of Marine Sciences, University of the Algarve, Faro, Portugal; 5-7.

Janssen Cl, Kiliaan AJ. Long-chain polyunsaturated fatty acids (LCPUFA) from genesis to senescence: The influence of LCPUFA on neural development, aging, and neurodegeneration. Prog Lipid Res. 2014;53:1-17.

Carolsfeld J, Powell JF, Park M, Fischer WH, Craig AG, Chang JP, Rivier JE, Sherwood NM. Primary structure and function of three gonadotropin-releasing hormones, including a novel form, from an ancient teleost, herring. Endocrinol. 2000;141:505-512.

Carrera M, Cañas B, Gallardo JM. Proteomics for the assessment of quality and safety of fishery products. Food Res Int. 2013;54(1):972-979.

Carrillo Estévez Manuel Adrián. 2009. Libro la reproducción de los peces: aspectos básicos y sus aplicaciones en acuicultura, Madrid España. Editorial Fundación Observatorio Español de Acuicultura (c) CSIC, 260-270.

CCI (Corporación Colombiana Internacional, Sistemas de información de precios). 2009.Número 8, Volumen 5

Cejas JR, Almansa E, Jerez S, Bolaños A, Samper M, Lorenzo A. Lipid and fatty acid composition of muscle and liver from wild and captive mature female broodstocks of white sea bream, Diplodus sargus. Comp Biochem Physiol. 2004;138:91-102.

Chapman SC, Sawitzke AL, Campbell DS, Schoenwolf GC. A threedimensional atlas of pituitary gland development in the zebrafish. J Comp Neurol. 2005;487: 428-440.

Chen CC, Fernald RD. GnRH and GnRH receptors: distribution, function and evolution. J Fish Biol. 2008;73:1099-1120.

Constantinos C, Mylonas, Fostier Al, Zanuy S. Broodstock management and hormonal manipulations of fish reproduction, Gen Comp Endocrinol. 2010;165:516-534.

Corinne L. Bloch, Noa Kedar, Matan Golan, Michael J. Gutnick, Ilya A. Fleidervish, Berta Levavi-Sivan. Long-term GnRH-induced gonadotropin secretion in a novel hypothalamo-pituitary slice culture from tilapia brain, Gen Comp Endocrinol. 2014;207(1):21-27.

Counis R, Laverriere JN, Garrel G, Bleux C, Cohen-Tannoudji J, Lerrant $\mathrm{Y}$, Kottler ML, Magre S. Gonadotropin-releasing hormone and the control of gonadotrope function. Reprod Nutr. 2005;45:243-254. 
Coward K, Little DC. Culture of the 'aquatic chicken'. Present concerns and future prospects. Biologist. 2001;48:12-16.

Dunning KR, Robker RL. Promoting lipid utilization with I-carnitine to improve oocyte quality. Anim Reprod Sci. 2012;134:69-75.

El-Sayed AFM, Kawanna M. Effects of dietary protein and energy levels on spawning performance of Nile tilapia (Oreochromis niloticus) broodstock in a recycling system. Aquaculture. 2008;280:179-184.

FAO. The State of World Fisheries and Aquaculture. 2014. Food and Agriculture Organization of the United Nations, Rome; 223.

FAO. The State of World Fisheries and Aquaculture. 2012. Food and Agriculture Organization of the United Nations, Rome, Italy.

Fonseca-Madrigal J, Karalazos V, Campbell PJ, Bell JG, Tocher DR. Influence of dietary palm oil on growth, tissue fatty acid compositions, and fatty acid metabolism in liver and intestine in rainbow trout (Oncorhynchus mykiss). Aquacult. Nutr. 2005;11:241-250.

Frisen L. Rapid transmandibular hypophysectomy of small fish Experientia. 1967; 23: 1079-1081.

Furuita H, Hori K, Suzuki, Sugita T, Yamamoto T. Effect of $n-3$ and $\mathrm{n}-6$ fatty acids in broodstock diet on reproduction and fatty acid composition of broodstock and eggs in the Japanese eel AnguiIla japonica. Aquaculture. 2007;267:55-61.

Furuita $\mathrm{H}$, Yamamoto $\mathrm{T}$, Shima T, Suzuki N, Takeuchi T. Effect of arachidonic acid levels in broodstock diet on larval and egg quality of Japanese flounder Paralichthys olivaceus. Aquaculture. 2003;220:725-735.

Garrel G, Simon V, Denoyelle C, Cruciani-Guglielmacci C, Migrenne S, Counis R, Magnan C, Cohen-Tannoudji J. Unsaturated fatty acids stimulate LH secretion via novel PKCepsilon and -theta in gonadotrope cells and inhibit GnRH-induced LH release. Endocrinol. 2011;152:3905-3916.

Gonzalez-Martinez D, Zmora N, Mananos E, Saligaut D, Zanuy S, Zohar Y, Elizur A, Kah O, Munoz-Cueto JA. Immunohistochemical localization of three different prepro-GnRHs in the brain and pituitary of the European sea bass (Dicentrarchus labrax) using antibodies to the corresponding $\mathrm{GnRH}$-associated peptides J Comp Neurol. 2002;446: 95-113.

Roch GJ, Busby ER, Sherwood NM. Evolution of GnRH: Diving deeper. Department of Biology, University of Victoria. Gen Comp Endocrinol. 2011;171:1-16.

Gulliver CE, Friend MA, King BJ, Clayton EH. The role of omega-3 polyunsaturated fatty acids in reproduction of sheep and cattle, Animal Reproduction Sci. 2012;131:9-22.

Guzmán JM, Ramos J, Mylonas CC, Mañanós EL. Spawning performance and plasma levels of $\mathrm{GnRHa}$ and sex steroids in cultured female Senegalese sole (Solea senegalensis) treated with different GnRHa-delivery systems. Aquacult. 2009;291:200-209.

Hachero Lobaccaro JM, Gallot D, Lumbroso S, Mouzat K. Liver X receptors and female reproduction: when cholesterol meets fertility. J Endocrinol Invest. 2013;36: 55-60.
Hachero-Cruzado I, Olmo P, Sánchez B, Herrera M, Domingues P. The effects of an artificial and a natural diet on growth, survival and reproductive performance of wild caught and reared brill (Scophthalmus rhombus). Aquacult. 2009;291:82-88.

Hildahl J, Sandvik GK, Edvardsen RB, Fagernes C, Norberg B, Haug $\mathrm{TM}$, Weltzien FA. Identification and gene expression analysis of three $\mathrm{GnRH}$ genes in female Atlantic cod during puberty provides insight into GnRH variant gene loss in fish, Gen Comp Endocrinol. 2011;172:458-467.

Hildahl J, Sandvik GK, Edvardsen RB, Fagernes C, Norberg B, Haug TM, Weltzien FA. Identification and gene expression analysis of three $\mathrm{GnRH}$ genes in female Atlantic cod during puberty provides insight into GnRH variant gene loss in fish, Gen Comp Endocrinol. 2011;172:458-467.

Hodne K, Strandabø RA, von Krogh K, Nourizadeh-Lillabadi R, Sand O, Weltzien FA, Haug T.M. Electrophysiological differences between fshb- and lhb-expressing gonadotropes in primary culture. Endocrinol. 2013;154:3319-3330.

Howell B, Pricket R, Cañavate P, Mañanos E, Dinis MT, Conceição L, Valente L. 2011. Report of the 5th Workshop on the Cultivation of Soles, Centre of Marine Sciences (CCMAR), University of the Algarve, Faro, Portugal; 12.

Imanaga Y, Nyuji M, Amano i, Takahashi A, Kitano H, Yamaguchi A, Matsuyama M. Characterization of gonadotropin-releasing hormone and gonadotropin in jack mackerel (Trachurus japonicus): Comparative gene expression analysis with respect to reproductive dysfunction in captive and wild fish. Aquacult. 2014;(428-429):226-235.

Izquierdo MS, Fernandez-Palacios H, Tacon AGJ. Effect of broodstock nutrition on reproductive performance of fish. Aquacult. 2001;197:25-42.

Jackson AJ, Shepherd CJ. The future of fishmeal and fish oil. Ryder J, Ababouch L, Balaban M. 2012. Second International Congress on Seafood Technology on Sustainable, Innovative and Healthy Seafood. Pp 18-21

Jalabert B. Particularities of reproduction and oogenesis in teleost fish compared to mammals Reprod Nutr. 2005;45:261-279.

Jaya-Ram A, Kuah M, Lim P, Kolkovski S, Shu-Chien A. Influence of dietary HUFA levels on reproductive performance, tissue fatty acid profile and desaturase and elongase mRNA expression in female zebrafish Danio rerio. Aquacult. 2008; 277:275-281.

Jump DB, Thelen A, Ren B, and Mater M. Multiple mechanisms for polyunsaturated fatty acid regulation of hepatic gene transcription. Prostaglandins Leukotrienes Essent. Fatty Acids. 1999;60:345-349.

Jump DB. The biochemistry of n-3 polyunsaturated fatty acids. J Biol Chem. 2002; 277:8755-8758.

Karapanagiotidis IT, Bell M, Little D, Yakupitiyage A. Replacement of dietary fish oils by alpha-linolenic acid-rich oils lowers omega 3 content in tilapia flesh. Lipids. 2007;42:547-559.

Levavi-Sivan B, Avitan A, Kanias T. Characterization of the inhibitory dopamine receptor from the pituitary of tilapia. Fish Physiol Biochem. 2003;28:73-75. 
Levavi-Sivan B, Bloch CL, Gutnick MJ, Fleidervish IA. Electrotonic coupling in the anterior pituitary of a teleost fish. Endocrinol. 2005;146:1048-1052.

Levavi-Sivan B, Avitan A, Kanias T. Characterization of the inhibitory dopamine receptor from the pituitary of tilapia. Fish Physiol Biochem. 2003;28:73-75.

Levavi-Sivan B, Bogerd J, Mañanós EL, Gómez A, Lareyre JJ. Perspectives on fish gonadotropins and their receptors. Gen Comp Endocrinol. 2010;165:412-437.

Levavi-Sivan B, Safarian H, Rosenfeld H, Elizur A, Avitan A. Regulation of gonadotropin-releasing hormone $(\mathrm{GnRH})$ receptor gene expression in tilapia: effect of $\mathrm{GnRH}$ and dopamine. Biol Reprod. 2004;70:1545-1551.

Ling S, Kuah MK, Muhammad TST, Kolkovski S, Shu-Chien AC. Effect of dietary HUFA on reproductive performance, tissue fatty acid profile and desaturase and elongase mRNA in female swordtail Xiphpphorus helleri. Aquacult. 2006;261:204-214.

Lund I, Steenfeldt SJS, Banta G, Hansen BW. The influence of dietary concentrations of arachidonic acid and eicosapentaenoic acid at various stages of larval ontogeny on eye migration, pigmentation and prostaglandin content of common sole larvae (Solea solea L.). Aquacult. 2008;276:143-153.

Lupatsch I, Deshev R, Magen I. Energy and protein demands for optimal egg production including maintenance requirements of female tilapia Oreochromis niloticus. Aquacult. 2010;41:763769.

Mañanos E, Duncan NJ, Mylonas CC, Cabrita E, Robles V, Herraez P. 2008Reproduction and control of ovulation, spermiation and spawning in cultured fish, and Methods in Reproductive Aquaculture. Pp. 1-78.

Golan M, Levavi-Sivan B. Social dominance in tilapia is associated with gonadotroph hyperplasia. Gen Comp Endocrinol. 2013;192(1):126-135.

Mateos GG, Rebollar PG, Medel P. 1999. Utilización De Grasas Y Productos Lipídicos En Alimentación Animal: Grasas Puras Y Mezclas. Departamento De Producción Animal. Universidad Politécnica De Madrid.

Mateos GG, Rebollar PG, Medel P. 1999. Utilización De Grasas Y Productos Lipídicos En Alimentación Animal: Grasas Puras Y Mezclas. Departamento De Producción Animal. Universidad Politécnica De Madrid.

Mazorra C, Bruce JG, Bell A, Davie E, Alorend N, Jordan JF, et al. Dietary lipid enhancement of broodstock reproductive performance and egg and larval quality in Atlantic halibut (Hippoglossus hippoglossus). Aquacult. 2003;227(1-4):21-33.

Meunpol O, Meejing P, Piyatiratitivorakul S. Maturation diet based on fatty acid content for male Penaeus monodon (Fabricius) broodstock. Aquacult. Research, 2005;36:1216-1225.

Mittelholzer C, Andersson E, Taranger GL, Consten D, Hirai T, SenthiIkumaran B, et al. Molecular characterization and quantification of the gonadotropin receptors FSH-R and LH-R from Atlantic cod (Gadus morhua). Gen Comp Endocrinol. 2009;160(1):4758. doi: 10.1016/j.ygcen.2008.10.015.
Miyamoto K, Hasegawa Y, Minegishi T, Nomura M, Takahashi Y, Igarashi $\mathrm{M}$, et al. Isolation and characterization of chicken hypothalamic luteinizing hormone-releasing hormone. Biochem Biophys Res Commun. 1982;107(3):820-827.

Moberg G, Watson J, Doroshov S, Papkoff H, Pavlick R. Physiological evidence for two sturgeon gonadotrophins in Acipenser transmontanus. Aquacult. 1995;135(1-3):27-39.

Morais S, Mendes AC, Castanheira MF, Coutinho J, Bandarra N, Dias J, et al. New formulated diets for Solea senegalensis broodstock: Effects of parental nutrition on biosynthesis of long-chain polyunsaturated fatty acids and performance of early larval stages and juvenile fish. Aquacult. 2014;432:374-382. https://doi. org/10.1016/j.aquaculture.2014.04.033.

Muskiet FAJ, Van Goor SA, Kuipers RS, Velzing-Aarts FV, Smit EN, Bouwstra HJanneke, et al. Long-chain polyunsaturated fatty acids in maternal and infant nutrition. Prostaglandins Leukot Essent Fat Acids. 2006;75(3):135-144.

Navarro RD, Navarro FK, Seixas, Filho JT, Ribeiro Filho. Nutrição e alimentação de reprodutores de peixes. Revista Augustus. 2010;30:108-118.

Navarro RD, Pereira Navarro FK, Ribeiro Filho OP, Motta Ferreira, Pereira Maia M, Seixas Filho JT. Quality of polyunsaturated fatty acids in Nile tilapias (Oreochromis niloticus) fed with vitamin $\mathrm{E}$ supplementation. Food Chem. 2010;134(1):215-218.

Norambuena F, Estevez A, Bell G, Carazo I, Duncan N. Proximate and fatty acid compositions in muscle, liver and gonads of wild versus cultured broodstock of Senegalese sole (Solea senegalensis), Aquacult. 2012;356-357:176-185.

NRC. 2011. Nutrient requirements of fish and shrimp. The National Academies Press, Washington, D.C. 228

Ogawa S, Akiyama G, Kato S, Soga T, Sakuma Y, Parhar IS. Immunoneutralization of gonadotropin-releasing hormone type-III suppresses male reproductive behavior of cichlids. Neurosci Lett. 2006;403(3):201-205. DOI: 10.1016/j.neulet.2006.02.041

Okuzawa K. Puberty in teleosts. Fish Physiol Biochem. 2002;26(1):3141.

Orozco J. 2012. Análisis del sector piscícola para la generación de estrategias enfocadas en la exportación a Estados Unidos de tilapia procesada producida en el Huila. Trabajo de grado. Universidad de Rosario.

Padmanabhan V, McNeilly AS. Is there an FSH-releasing factor. Reproduction. 200;121(1):21-30.

Levkowitz G, Zohar Y, Gothilf Y. Nasal embryonic LHRH factor plays a role in the developmental migration and projection of gonadotropin-releasing hormone 3 neurons in zebrafish. Dev Dyn. 2009;238(1):66-75.

Pérez P, Vieira P, Aparecida de Jesus P, Carvalho A, Solis L, Fonseca $R$. Efeito do uso de óleo na dieta sobre a lipogênese e o perfil lipídico de tilápias-do-nilo. Rev Bras Zootec. 2008;37:1331. 1337.

Pickova J, Brannas E, Andersson T. Importance of fatty acids in broodstock diets with emphasis on Arctic char (Salvelinus alpinus) eggs. Aquacult Internat. 2007;15: 305-311. 
Planas JV, Swanson P. Maturation-associated changes in the response of the salmon testis to the steroidogenic actions of gonadotropins (GtH-I and GtH-II) in vitro. Biol Reprod. 1995;52(3):697704.

Pogoda HM, Hammerschmidt M. Molecular genetics of pituitary development in zebrafish. Semin Cell Dev Biol. 2007;18(4):543558.

Pohlenz C, Delbert M, Gatlin III. Interrelationships between fish nutrition and health. Aquacult. 2014;431:111-117

Polakof S, Médale F, Skiba-Cassy S, Corraze G, Panserat S. Molecular regulation of lipid metabolism in liver and muscle of rainbow trout subjected to acute and chronic insulin treatments Domest. Anim Endocrinol. 2010;39(1):26-33.

Querat B, Sellouk A, Salmon C. Phylogenetic analysis of the vertebrate glycoprotein hormone family including new sequences of sturgeon (Acipenser baeri) beta subunits of the two gonadotropins and the thyroid-stimulating hormone. Biol Reprod. 2000;63(1):222-228.

Rocha A, Gomez A, Zanuy S, Cerda-Reverter JM, Carrillo M. Molecular characterization of two sea bass gonadotropin receptors: cDNA cloning, expression analysis, and functional activity. Mol Cell Endocrinol. 2007;272(1-2):63-76.

Rodrígues PM, Tomé SS, Dias J, Jessen F. PROTEOMICS in aquaculture: Applications and trends. J Proteom. 2012;75(14):43254345.

Sawanboonchun J. 2009. Atlantic Cod (Gadus morhua L.) Broodstock Nutrition: The role of arachidonic acid and astaxanthin as determinants of egg quality $\mathrm{PhD}$ dissertation, University of Stirling, Institute of Aquaculture, Stirling; 212.

Schulz, RW, França, LR, Lareyre JJ, LeGac F, Chiarini-Garcia H, Nobrega R, Miura T. Spermatogenesis in fish. Gen Comp Endocrinol. 2010;165(3):390-411.

Seiliez I, Panserat S, Corraze G, Kaushik S, Bergot P. Cloning and nutritional regulation of a $\Delta 6$-desaturase-like enzyme in the marine teleost gilthead seabream (Sparus aurata). Comp Biochem Physiol. 2003;135:449-460.

Sharma TP, Nett TM, Karsch FJ, Phillips DJ, Lee JS, Herkimer C, Padmanabhan V. Neuroendocrine control of FSH secretion. IV. Hypothalamic control of pituitary FSH-regulatory proteins and their relationship to changes in $\mathrm{FSH}$ synthesis and secretion. Biol Reprod. 2012;86(6):1-171.

Shepherd CJ, Jackson AJ. Global fishmeal and fish-oil supply: inputs, outputs and markets. J Fish Biol. 2013;83(4):1046-1066.

Sherwood N, Eiden L, Brownstein M, Spiess J, Rivier J, Vale W. 1983. Characterization of a teleost gonadotropin-releasing hormone. Proc Natl Acad Sci. USA. 1983;80(9):2794-2798.

Simopoulos AP. Evolutionary aspects of diet: the omega-6/omega-3 ratio and the brain. Mol Neurobiol. 2011;44:203-215.

Sorbera A, Asturiano JF, Carrillo M, Zanuy. Effects of polyunsaturated fatty acids and prostaglandins on oocyte maturation in a marine teleost, the European sea bass (Dicentrarchus labrax). Biol Reprod. 2001;64:382-389.
Suárez E. 2011. Estrategia para la implementación de Buenas Prácticas de Producción Acuícola (BPPA) en agroempresas productoras de tilapia del departamento del Huila. Tesis de Maestría. Universidad Nacional de Colombia Bogotá.

Subhadra B, Lochmann R, Rawles S, Chen R. Effect of dietary lipid source on the growth, tissue composition and hematological parameters of largemouth bass (Micropterus salmoides). Aquacult. 2006;255(1-4):210-222.

Swanson P, Suzuki K, Kawauchi H, Dickhoff WW. Isolation and characterization of two coho salmon gonadotropins, GtH-I and GtH-II. Biol Reprod. 1991;44(1):29-38.

Tacon AGJ, Metian M. Fish matters: importance of aquatic foods in human nutrition and global food supply. Rev Fish Sci. 2013;21:22-38.

Tacon AGJ, Metian M. Global overview on the use of fish meal and fish oil in industrially compounded aquafeeds: trends and future prospects. Aquacult. 2008;285(1-4):146-158.

Tacon P, Baroiller JF, Le Bail PY, Prunet P, Jalabert B. Effect of egg deprivation on sex steroids, gonadotropin, prolactin, and growth hormone profiles during the reproductive cycle of the mouthbrooding cichlid fishOreochromis niloticus. Gen Comp Endocrinol. 2000;117(1):54-65.

Taranger GL, Carrillo M, Schulz RW, Fontaine P, Zanuy S, Felip A, et al. Control of puberty in farmed fish. Gen Comp Endocrinol. 2010;165(3):483-515.

Teoh Chaiw-Yee, Turchini Giovanni M, Wing-Keong Ng. Erratum to "Genetically improved farmed Nile tilapia and red hybrid tilapia showed differences in fatty acid metabolism when fed diets with added fish oil or a vegetable oil blend". Aquacult. 2011;316(1-4):144-154.

Thackray VG, Mellon PL, Coss D. Hormones in synergy: regulation of the pituitary gonadotropin genes. Mol Cell Endocrinol. 2010;314:192-203.

Thompson IR, Kaiser UB. GnRH pulse frequency-dependent differential regulation of $\mathrm{LH}$ and $\mathrm{FSH}$ gene expression. Mol Cell Endocrinol. 2014;85(1-2):28-35

Tocher DR, Fonseca-Madrigal J, Bell JG, Dick JR, Henderson RJ, Sargent JR. Effects of diets containing linseed oil on fatty acid desaturation and oxidation in hepatocytes and intestinal enterocytes in Atlantic salmon (Salmo salar). Fish Physiol Biochem. 2002;26:157-170.

Tocher DR. Fatty acid requirements in ontogeny of marine and freshwater fish. Aquacult Res. 2010;41:717-732.

Tocher DR. Metabolism and functions of lipids and fatty acids in teleost fish. Rev Fish Sci. 2003;11(2):107-184.

TradeMap. Internacional Trade Centre. Estadísticas de comercio para el desarrollo internacional de las empresas. (2012) www. trademap.org.

Turchini GM, Ng WK, Tocher DR. 2011. Fish Oil Replacement and Alternative Lipid Sources in Aquaculture Feeds, Taylor \& Francis, CRC Press, Boca Raton; 533. 
Turchini GM, Torstensen BE. Ng WK. 2009. Fish oil replacement in finfish nutrition. Rev Aquacult. 2009;1(1):10-57.

Vassallo-Agius R, Watanabe T, Yoshizaki G, Satoh S, Takeuchi T. Quality of eggs and spermatozoa of rainbow trout fed an n-3 essential fatty acid-deficient diet and its effects on the lipid and fatty acid components of eggs, semen and livers. Fish Sci. 2001;67(5):818-827.

Volkoff H, Xu MY, MacDonald E, Hoskins L. Aspects of the hormonal regulation of appetite in fish with emphasis on goldfish, Atlantic cod and winter flounder: notes on actions and responses to nutritional, environmental and reproductive changes. Comp Biochem Physiol A Mol Integr Physiol. 2009;153(1):8-12.

Wade MG, Van Der Kraak G, Gerrits MF, Ballantyne JS. 1994. Release and steroidogenic action of polyunsaturated fatty acids in the goldfish testis. Biol Reprod.1994;51(1):131-139.

Watanabe T, Vassallo-Agius R. Broodstock nutrition research on marine finfish in Japan. Aquacult. 2003;227:35-61.

Wathes DC, Abayasekara DR, Aitken RJ. Polyunsaturated fatty acids in male and female reproduction. Biol Reprod. 2007;77:190201.

Weltzien FA, Andersson E, Andersen $\varnothing$, Shalchian-Tabrizi K, Norberg B. The brain-pituitary-gonad axis in male teleosts, with emphasis on the flatfish (Pleuronectiformes). Comp Biochem Physiol. 2004;137:447-477.

Weltzien Finn-Arne, Hildahl Jon, Hodne Kjetil, Okubo Kataaki, Haug Trude M. Embryonic development of gonadotrope cells and gonadotropic hormones - Lessons from model fish. Mol Cell Endocrinol. 2014;385:2-25.

Wilson R. Dietary effects of n-3 highly unsaturated fatty acid levels on egg and larval quality, and the fatty acid composition of the eggs of Chilean flounder Paralichthys adspersus broodstock. Aquacult Res. 2009;40(12):1400-1409.

Wing-Keong Ng, Cheong-Yew Chong, Yan Wang, Nicholas Romano. Effects of dietary fish and vegetable oils on the growth, tissue fatty acid composition, oxidative stability and vitamin E content of red hybrid tilapia and efficacy of using fish oil finishing diets. Aquacult. 2013;372-375:97-110

Wing-Keong $\mathrm{Ng}$, Yan Wang. Inclusion of crude palm oil in the broodstock diets of female Nile tilapia, Oreochromis niloticus, resulted in enhanced reproductive performance compared to broodfish fed diets with added fish oil or linseed oil. Aquacult. 2011;314:122-131.

Yaron Z, Gur G, Melamed P, Rosenfeld H, Elizur A, Levavi-Sivan B. Regulation of fish gonadotropins. Int Rev Cytol. 2003;225:131. 185.

Zanuy S, Carrillo M, Felip A, Rodriguez L, Blazquez M, Ramos J, Piferrer F. Genetic, hormonal and environmental approaches for the control of reproduction in the European sea bass (Dicentrarchus labrax L.). Aquacult. 2001;202:187-203.

Zohar Y, Muñoz-Cueto J, Elizur A, Kah O. Neuroendocrinology of reproduction in teleost fish. Gen Comp Endocrinol. 2010;165(3):438-455. 